\title{
LEISHMANIOSE VISCERAL (CALAZAR) NA ILHA DE SÃO LUÍS, MARANHÃO, BRASIL: EVOLUÇÃO E PERSPECTIVAS
}

\author{
Antonio Rafael da Silva, Graça Maria Castro Viana, Carlos Varonil, \\ Benedito Pires, Maria do Desterro S.D. Nascimento e Jackson M.L. Costa
}

\begin{abstract}
Leishmaniose visceral (calazar) entrou definitivamente como nosologia importante do Estado do Maranhão, Brasil, a partir de 1982. Desde então, vários autores têm trabalhado o tema do ponto de vista de relatos. No entanto, a parte de diagnóstico, tratamento e controle de cura percorreram caminhos difíceis e sempre preocupou os que estudam a doença que se instalou na Ilha de São Luís a partir da desestabilização dos ecótopos da Lutzomya longipalpis, o transmissor mais importante. Após 1993 a constatação de casos com má resposta a o antimoniato-n-metil glucamina (Glucantime®) veio se somar às outras preucupações. O estudo atual mostra como 0 Sistema Único de Saúde, através dos seus serviços, atua no controle da doença e conclui sobre a existência de refratariedade ao Glucantime ${ }^{\circledR}$ o que impõe maior vigilância no diagnóstico, tratamento e controle de cura dos pacientes.
\end{abstract}

Palavras-chaves: Leishmaniose visceral. Tratamento. Aspectos clínicos.

Em 1982, Silva e cols22 diagnosticaram quatro casos de calazar, no bairro do São Cristóvão. Seguem-se mais dois outros em São Bernardo, ambos bairros do município de São Luís (MA). $\mathrm{Na}$ seqüência, um surto com 32 casos, apresentado ao XIX Congresso da Sociedade Brasileira de Medicina Tropical inaugurou a história do calazar enquanto nosologia no Estado do Maranhão e sua urbanização no Brasil22. Nos anos seguintes, a doença atingiu os municípios de São José de Ribamar e Paço do Lumiar, sendo atualmente um dos maiores problemas de saúde pública da Ilha de São Luís, dada a freqüência com que vem acontecendo e o grupo populacional que mais acomete, os menores de 9 anos de idade21 2324 .

Estudos posteriores realizados pela Universidade Federal do Maranhão (UFMA) e Ministério da Saúde do Brasil, confirmam que o Maranhão juntamente com o Ceará, Bahia e Piauí, constituem os estados brasileiros com maiores prevalências de calazar na atualidade7 1517212327.

Em nosso meio, a invasão do calazar pode ser explicada: pelas condições geográficas, sobretudo climáticas representadas pela alta

Departamento de Patologia do Centro de Ciências da Saúde da Universidade Federal do Maranhão e Fundação Nacional de Saúde (FNS-MA), São Luís, MA, Brasil.

Endereço para correspondência: Dr.Antonio Rafael da Silva, Depto de Patologia/UFMA. Praça Madre Deus 2, 65025-560 São Luís, MA. Fax: (098) 222-5135;Tel: (098) 231-3644.

Recebido para publicação em 19/07/96. pluviosidade, que favorece biologicamente os criadouros de flebotomíneos. Outros fatores, tais como as profundas alterações que sofreu a Ilha de São Luís após o governo do Estado, à época, ceder extensas áreas para implantação dos pólos industriais da Companhia Vale do Rio Doce e da Indústria de Alumínio do Maranhão e, com isso, deslocando milhares de famílias que foram fazer assentamentos e se instalaram em condições inadequadas de saúde e saneamento 20.

A desestabilização dos ecótopos da Lutzomya longipalpis, o mais importante transmissor do calazar em nosso meio, e a concomitante mobilização da população canina, reservatório da Leishmania chagasi, completam a cadeia epidemiológica de uma doença que se tornou endêmica em solo maranhense, a partir dessa época3 8162427.

Embora o maior número de casos tenha ocorrido em dois momentos, 1984 e 1993, os acontecimentos relacionados com a doença, a partir de sua descoberta, passaram a ser problema à medida que se comprovaram situações que contribuíram não somente para o retardo do diagnóstico e tratamento, como também para manter a letalidade em níveis elevados.

Invariavelmente, os ambulatónios e enfermanias do Hospital Universitário (Unidades Presidente Dutra e Materno Infantil) e de outros hospitais, passaram a conviver com a doença e a assistir mortes provocadas pela mesma. De maneira expressiva, foi somente a partir de 1993 que uma variável preocupante se somou às outras: 
Silva AR, Viana GMC, Varonil C, Pires B, Nascimento MDSD, Costa JML Leishmaniose visceral (calazar) na Ilha de São Luís, Maranhão, Brasil: evolução e perspectivas. Revista da Sociedade Brasileira de Medicina Tropical 30:359-368, set-out, 1997.

a constatação de casos de calazar "não respondente", ou seja, que não apresentavam melhora clínica ou parasitária 28 ao antimoniato-N-metil-glucamina (Glucantime ${ }^{\circledR}$ ), a droga de escolha em nosso meio para seu tratamento 212325 .

Os fatos relatados orientaram a se proceder uma reavaliação da situação do calazar em nosso meio, colocando em foco se esta endemia é responsabilidade do Sistema Único de Saúde (SUS) por seus pressupostos de Universalização, Integralidade e Resolutividade da Assistência, ou se continua verticalizada, o que, a nosso ver gera descuidos e descompromissos.

É objetivo do presente trabalho, responder às questões abaixo formuladas tomando como base o ano de 1994: a) Quando e onde ocorreram os casos de calazar na Ilha de São Luís? b) Como são feitos o diagnóstico e tratamento? c) Onde são tratados e quais os critérios de cura? d) O que aconteceu aos pacientes após o tratamento? e) Existe refratariedade 28 ao tratamento?

\section{MATERIAL E MÉTODOS}

Trata-se de um estudo em pacientes diagnosticados, em tratamento e tratados nos hospitais, ambulatórios comunitários e nas unidades de saúde dos três municípios da Ilha de São Luís (São Luís, São José de Ribamar e Paço do Lumiar) e se deu em dois momentos: o primeiro constou do registro contido na ficha de notificação de todos os pacientes que tiveram diagnóstico de calazar, seguido de tratamento, em 1994; o segundo de um estudo retrospectivo, por amostragem, realizado nos meses de julho e agosto de 1995 e se deu em função do exame laboratorial específico que confirmou o diagnóstico. Contou com a participação de um professor de Doenças Infecciosas e Parasitárias, estudantes de medicina, técnicos e funcionários da FNS, conhecedores das áreas e dos pacientes.

A amostragem constou de tres subgrupos de 72 pacientes que tiveram diagnóstico confirmado pela punção medular (PM), pela imunofluorescência (IFI) e pelo mielograma mais imunofluorescência (PM + IFI). Devido a importância que se deu aos óbitos a equipe decidiu universalizar, ou seja, registrar as informações obtidas sobre os mesmos.

Os pacientes foram vistos e examinados em suas habitações em localidades situadas nas periferias de bairros, em pequenos logradouros, em sítios e basicamente em invasões. A visita às localidades (Figura 1) serviram para registros: epidemiológico, com o objetivo de confirmar o caso de calazar na localidade, identificar a qualidade de moradia e a situação de trabalho da família onde ocorreu a doença; clínico, baseado em anamnese retrospectiva para confirmação de dados compatíveis com o quadro clínico de calazar e na realização de exame físico atual que objetiva identificar a doença em atividade, tomando como parâmetro a existência de febre e esplenomegalia, além dos achados de palidez cutâneo-mucosa, emagrecimento, baixo peso e fígado palpável. O exame físico realizou-se com o paciente em decúbito dorsal e a palpação abdominal em inspiração profunda e o baço anotado segundo os graus I, II, III, IV e V baseado na classificação de Hackett13, 1954; terapêutico para confirmação do medicamento utilizado, a dose, a duração, a via de administração, o local de tratamento, bem como a existência de tratamentos posteriores à alta e, finalmente, 0 controle de cura realizado após o tratamento que serviu como orientação para a alta.

Os casos encontrados com sinais e sintomas indicativos de suspeita de calazar em atividade foram registrados para acompanhamento e posterior avaliação diagnóstica.

\section{RESULTADOS}

O primeiro momento do estudo mostra que o calazar ocorreu em todos os meses do ano de 1994 (Figura 2), nos três municípios da Ilha de São Luís (MA), sendo $135(41,4 \%)$ em São Luís, 125 (38,3\%) em São José de Ribamar e 66 $(22,2 \%)$ em Paço do Lumiar, perfazendo 326 casos. O sexo masculino foi o mais acometido, 190 casos (58,3\%). Quanto à faixa etária, 79,7\% eram menores de 9 anos, sendo $93(28,5 \%)$ de 0 a 23 meses, $105(32,2 \%)$ entre 2 e 4 anos e $62(19,0 \%)$ entre 5 e 9 . Na faixa de 10 a 14 anos, ocorreram 10 casos $(3,1 \%)$ e $56(17,2 \%)$ acima de 15 anos de idade. 0 paciente mais jovem tinha 6 meses e o mais velho 72 anos. 0 registro, contido nas fichas de notificação, catalogou 13 óbitos dos quais 12 sob regime de internação.

No segundo momento, no estudo de campo, foram visitadas 128 famílias nas 86 localidades e identificados 152 pacientes. Destes, 130 $(85,6 \%)$, sendo a maioria do sexo masculino, submeteram-se à anamnese e exame físico. Os 
Silva AR, Viana GMC, Varonil C, Pires B, Nascimento MDSD, Costa JML Leishmaniose visceral (calazar) na Ilha de São Luís, Maranhão, Brasil: evolução e perspectivas. Revista da Sociedade Brasileira de Medicina Tropical 30:359-368, set-out, 1997.
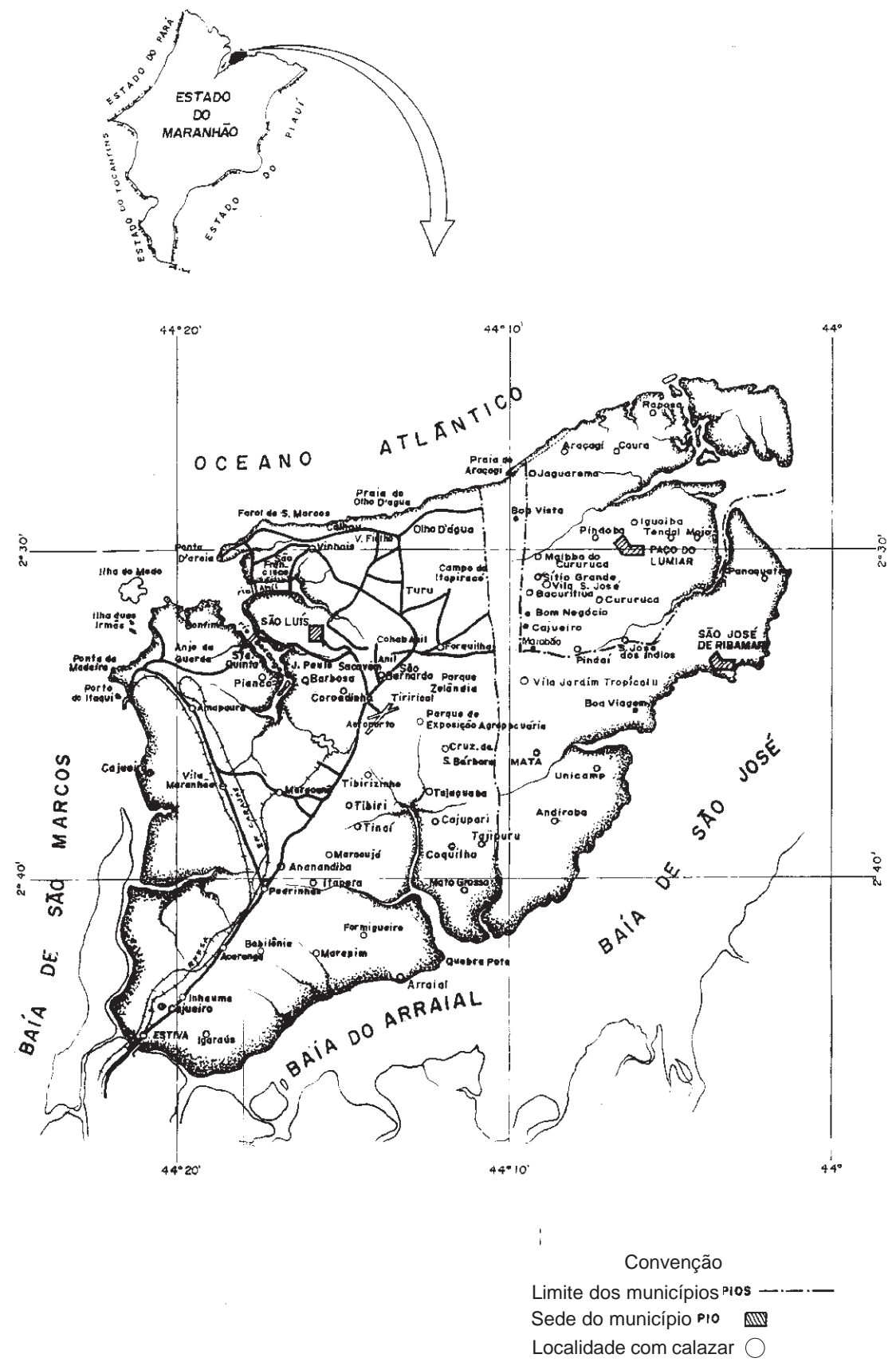

Figura 1 - Mapa da Ilha de São Luís.

outros $22(14,5 \%)$ já haviam falecido, sendo que 9 deles identificados durante as visitas de campo. Deixaram de ser encontradas as famílias de 64 pacientes devido a mudança de endereço ou migrações para outros municípios do Estado.
Os achados que se seguem nas Tabelas $1 \mathrm{e}$ 2 e Figura 3, referem-se a 130 casos, revistos no trabalho de campo. A Tabela 3 trata dos 22 casos de óbitos, que ocorreram em 1994.

A Tabela 1 confirma o achado anterior que demonstra ser os menores de 2 a 4 anos, com 
Silva AR, Viana GMC, Varonil C, Pires B, Nascimento MDSD, Costa JML Leishmaniose visceral (calazar) na Ilha de São Luís, Maranhão, Brasil: evolução e perspectivas. Revista da Sociedade Brasileira de Medicina Tropical 30:359-368, set-out, 1997.

$37,7 \%$, os mais acometidos dentre todos que tiveram confirmação laboratorial do diagnóstico de calazar.

\begin{tabular}{|c|c|c|c|c|c|}
\hline \multirow[t]{2}{*}{ Grupo etário } & \multicolumn{3}{|c|}{ Confirmação do diagnóstico } & \multirow[t]{2}{*}{ Total } & \multirow[t]{2}{*}{$\%$} \\
\hline & PM & IF] & $\mathrm{PM}+\mathrm{IFI}$ & & \\
\hline $10-23$ meses & 14 & 11 & 9 & 34 & 26,1 \\
\hline $2-4$ anos & 16 & 16 & 17 & 49 & 37,7 \\
\hline 5-9 anos & 6 & 11 & 11 & 28 & 21,5 \\
\hline 10-14 anos & - & 2 & 3 & 5 & 3,8 \\
\hline$>15$ anos & 3 & 4 & 7 & 14 & 10,8 \\
\hline Total & 39 & 44 & 47 & 130 & 100,0 \\
\hline
\end{tabular}

$\overline{\mathrm{PM}}=$ punção medular; IFI = imunofluorescência indireta.

\begin{tabular}{|c|c|c|c|c|}
\hline Exame físico & Fígado & $\begin{array}{c}\text { Freqüência } \\
\text { ajustada (\%) }\end{array}$ & Baço & $\begin{array}{c}\text { Freqüência } \\
\text { ajustada (\%) }\end{array}$ \\
\hline Impalpável & 77 & 59,3 & 86 & 66,2 \\
\hline Palpável & 53 & 40,7 & 44 & 33,8 \\
\hline Total geral & 130 & 100,0 & 130 & 100,0 \\
\hline
\end{tabular}

A anamnese retrospectiva colhida dos familiares e dos pacientes em condições de prestar informações confiáveis, confirmaram a presenca de sinais e sintomas compatíveis com a existếncia de um caso de calazar. A Figura 3 registra a ocorrência dos dados clínicos com os sintomas e os sinais mais freqüentemente referidos.

Embora os sintomas de febre, palidez, emagrecimento e aumento do volume abdominal liderem as queixas dos pacientes, na verdade foram os achados respiratórios (falta de ar e tosse) que mais motivaram os familiares a procurar os serviços de saúde e os médicos a pedir internação dos pacientes. Cefaléia foi um sintoma secundário bem como hiperpigmentação da pele (observadas poucas vezes) e escoriações da pele a maioria das vezes ligadas a quadro de escabiose.

Edema dos membros inferiores, mole, frio, indolor foi observado em mais de $30 \%$ dos

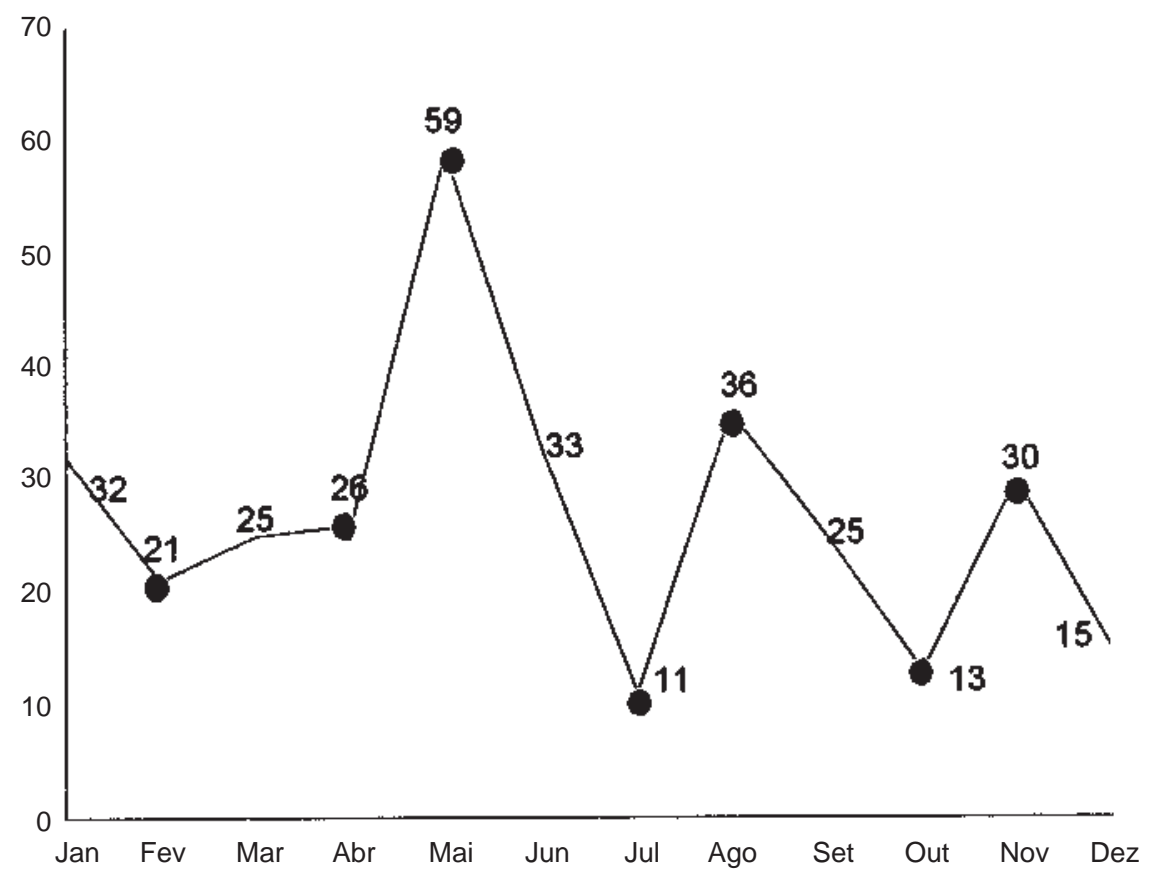

Figura 2 - Distribuição mensal dos casos de calazar na Ilha de São Luís, em 1994.

casos, em duas vezes acompanhado de anasarca. Quadros de icterícia quase sempre se acompanharam de gravidade, o que nem sempre aconteceu com hemorragias, principalmente nasais. Petéquias e equimoses foram raramente observadas. A queda de pêlos acompanhada de rarefação dos cabelos se relacionou com 0 tempo da doença e o grau de espoliação. 
Silva AR, Viana GMC, Varonil C, Pires B, Nascimento MDSD, Costa JML Leishmaniose visceral (calazar) na Ilha de São Luís, Maranhão, Brasil: evolução e perspectivas. Revista da Sociedade Brasileira de Medicina Tropical 30:359-368, set-out, 1997.

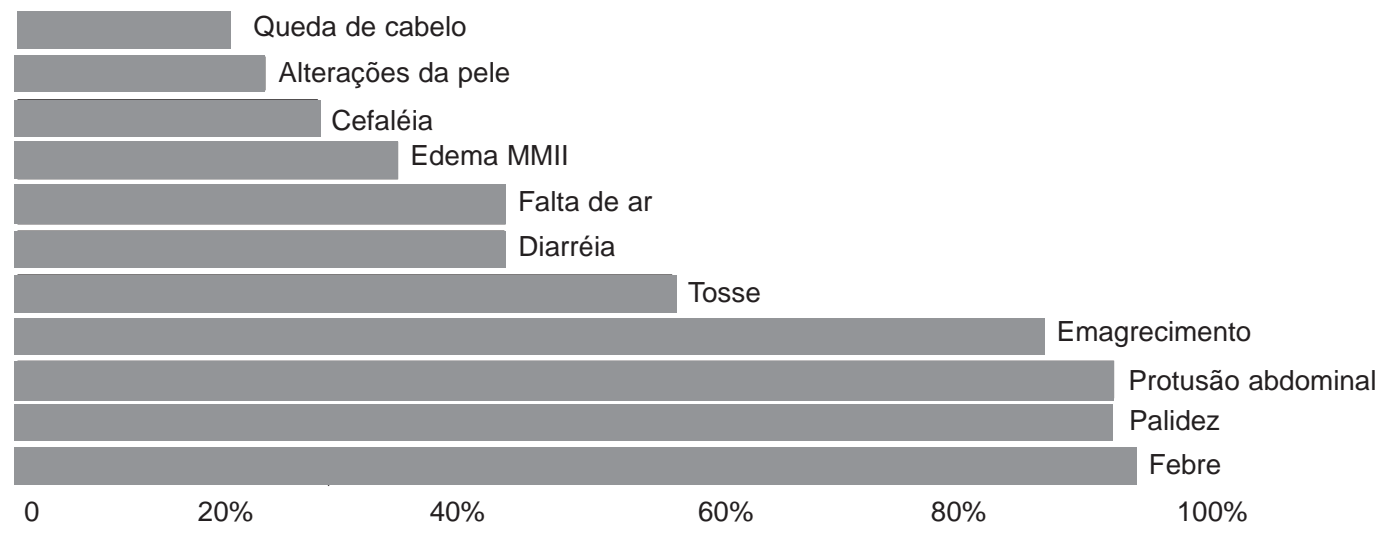

Figura 3 - Principais sinais e sintomas referidos quando da evolução realizada em 130 pacientes examinados no controle pós-tratamento de calazar na Ilha de São Luís-MA, em 1994.

Chamou a atenção o caráter irregular da febre, muitas vezes não sentida pelos pacientes e familiares que se surpreendiam quando a avaliação indicava estar acima de $38^{\circ} \mathrm{C}$.

Registra-se que a grande maioria dos pacientes, precisamente $121(93,0 \%)$, procurou mais de uma vez os ambulatórios, hospitais e postos de saúde, antes de receberem tratamento, fato que se deu em média no $3^{30}$ mês de doença. Alguns pacientes, a maioria adultos, tiveram evolução de 6 meses a 1 ano tendo-se internado com diagnóstico de pneumonia bacteriana, cirrose hepática, esquistossomose, desnutrição, malária, tuberculose, e outros.

O estado físico atual dos pacientes foi de regular para bom, encontrando-se 36 menores portadores de protusão abdominal, o que constitui achado comum nas crianças dessas localidades. Quanto à cor da pele e da mucosa conjuntival para estimativa da situação da série vermelha, 21 pacientes apresentavam mucosas hipocoradas de uma a duas cruzes numa referência de quatro. Quanto à temperatura, em 5 pacientes variou de $37,5^{\circ} \mathrm{C}$ a $38^{\circ} \mathrm{C}$ no momento do exame. Quanto aos outros aparelhos, estavam clinicamente normais não se encontrando sinais esteto-acústicos denunciadores de quaisquer patologias. Quanto à palpação abdominal, os resultados encontram-se na Tabela 2.

Todos os pacientes com baços palpáveis também apresentavam fígados palpáveis, constituindo o binômio hepato-esplenomegalia,
Quanto ao tamanho, $29(66,0 \%)$ apenas afloravam no gradil costal, grau I. Outros 10 $(22,7 \%)$ eram palpáveis até a linha horizontal esquerda que cruza a distância entre a cicatriz umbilical e o apêndice xifóide, grau II. Os 5 $(11,3 \%)$ restantes delimitavam a cicatriz umbilical, grau III, sendo considerados vantajosos para quem terminara o tratamento há mais de 6 meses. A Figura 4 mostra um dos pacientes.

$\mathrm{O}$ antimoniato- $\mathrm{N}$-metil-glucamina (glucantime ${ }^{\circledR}$ ) foi a droga de escolha em 125 pacientes e a via principal de administração foi a intramuscular. Como dado de certeza no Hospital Universitário a dose para cada paciente é de $20 \mathrm{mg} / \mathrm{Sb} / \mathrm{kg} /$ peso/dia, aplicados durante 20 dias. No Hospital Infantil a dose é a mesma, no entanto, o tratamento se dá em intervalos de 10 em 10 dias em duas ou três séries com o paciente internado. Nos outros hospitais existe uma variedade de conduta. Em 5 pacientes a droga foi anfotericina B lipossomal (Ambisome ${ }^{\circledR}$ ), utilizada num protocolo de pesquisa.

Quanto ao seguimento pós-tratamento, útil na definição da cura para alta, obteve-se informação que 86 pacientes receberam alta por iniciativa do próprio médico e 44 não retornaram às consultas de acompanhamento. Constatou-se a partir das informações, que somente o Hospital Universitánio e o Hospital Infantil davam sequência ao tratamento, acompanhando a evolução clínica e laboratorial até a "cura definitiva, que teve como parâmetros: ausência de sintomas, retorno do baço e do 
Silva AR, Viana GMC, Varonil C, Pires B, Nascimento MDSD, Costa JML Leishmaniose visceral (calazar) na Ilha de São Luís, Maranhão, Brasil: evolução e perspectivas. Revista da Sociedade Brasileira de Medicina Tropical 30:359-368, set-out, 1997.

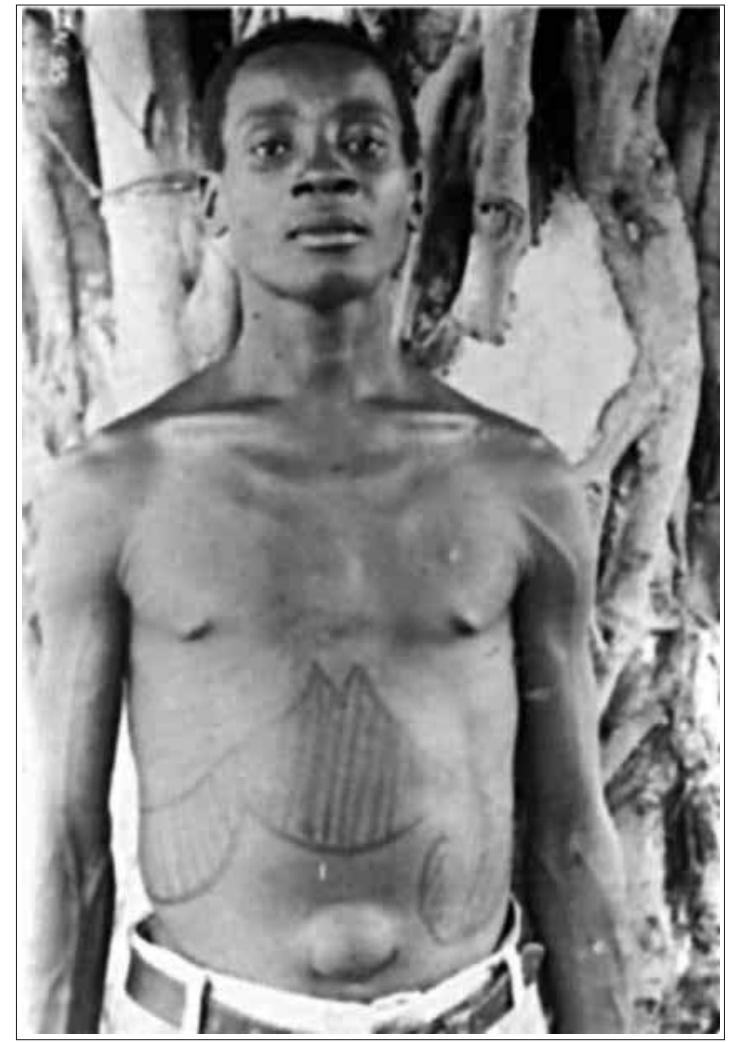

Figura 4 - J.R.S, 35 anos, masculino morador da Coheb do Sacavém, com hepatoesplen omegalia e manchas escuras no tronco, 60 dias após uso de antimoniato de N-metil glucamina (glucantime) na dose de $20 \mathrm{mg} / \mathrm{kg} / \mathrm{peso} / \mathrm{dia}$ durante 20 dias.

fígado aos padrões de normalidade, normalização do hemograma, da velocidade de hemossedimentação e do proteinograma, com retorno ao padrã̃o normal albumina/globulina. O tempo de observação dos pacientes foi variável. No ambulatório do Hospital Universitário (Unidade Presidente Dutra) foi em torno de 6 meses.
Em 1994 o registro baseado na ficha de notificação apontou para 13 mortes. 0 trabalho de campo permitiu identificar mais 9 óbitos não notificados. Dessa maneira ocorreram 22 óbitos, dos quais 16 durante o tratamento, 4 após terem alta hospitalar e 2 que não chegaram a realizar tratamento. A Tabela 3 distingue os óbitos, chamando-se a atenção que 13 ocorreram na faixa etánia de 6 a 23 meses. Do total de óbitos, 14 (63,6\%) pertenciam ao sexo masculino.

Quanto ao local de ocorrência dos óbitos, a mesma tabela mostra que todos os pacientes que faleceram tiveram confirmado o diagnóstico, ll por punção medular, 5 por imunofluorescência indireta e 6 por punção medular + imunofluorescência indireta.

Dentre os que morreram um era portador de Esquistossosmose mansoni, estava no 20응 dia de tratamento com antimonial e com estado geral bastante melhorado. Três horas após tomar a última dose de glucantime, apresentou dor abdominal, inquietação, crise de choro para em seguida ser encontrado morto. Este paciente, de 23 anos, foi recebido no ambulatório por apresentar quadro de insuficiência cardíaca e, além da hepatomegalia, apresentava esplenomegalia grau III. Tratado com diuréticos e digitálicos melhorou do quadro e, só após, submeteu-se a diagnóstico de calazar seguido de tratamento. Outro de 30 anos, esteve internado com diagnóstico de tuberculose e, após ter confirmado o diagnóstico de calazar, faleceu.

Dos 5 pacientes que tiveram diagnóstico confirmado pela IFI, 2 deles internaram-se por apresentar quadro de dispnéia e derrame pleural; um deles recebeu alta e o outro evoluiu com insuficiência renal aguda, chegando ao óbito por sangramento considerável de vasos catéter para diálise peritoneal. Os demais, 2 faleceram nas primeiras 12 horas de internação e do terceiro não se obteve informações.

Tabela 3 - Distribuição dos óbitos de calazar em pacientes que foram tratados na Ilha de São Luís, em 1994.

\begin{tabular}{|c|c|c|c|c|c|c|c|c|c|}
\hline \multirow[t]{2}{*}{ Faixa etária } & \multicolumn{2}{|c|}{ Sexo } & \multicolumn{3}{|c|}{ Local dos óbitos } & \multicolumn{3}{|c|}{ Confirmação do diagnóstico } & \multirow[t]{2}{*}{ Total } \\
\hline & masc & fem & $\mathrm{H}$ & $\mathrm{R}$ & SI & PM & IFI & $\mathrm{PM}+\mathrm{IFI}$ & \\
\hline$<6$ meses & - & - & - & - & - & - & - & - & - \\
\hline $6-23$ meses & 6 & 7 & 10 & - & 3 & 7 & 1 & 5 & 13 \\
\hline $2-4$ anos & 3 & 1 & 1 & - & 3 & 3 & 1 & - & 4 \\
\hline $5-9$ anos & 2 & - & - & 1 & 1 & 1 & 1 & - & 2 \\
\hline $10-14$ anos & - & - & - & - & - & - & - & - & - \\
\hline 15 anos & 3 & - & 2 & 1 & - & - & 2 & 1 & 3 \\
\hline Total & 14 & 8 & 13 & 2 & 7 & 11 & 5 & 6 & 22 \\
\hline
\end{tabular}

$\mathrm{H}$ = hospital; $\mathrm{R}$ = residência; $\mathrm{SI}$ = sem informação. 
Silva AR, Viana GMC, Varonil C, Pires B, Nascimento MDSD, Costa JML Leishmaniose visceral (calazar) na Ilha de São Luís, Maranhão, Brasil: evolução e perspectivas. Revista da Sociedade Brasileira de Medicina Tropical 30:359-368, set-out, 1997.

Sobre as localidades visitadas com exceção de Divinéia, Coheb do Sacavém, e Cidade Operária, que são bairros, Jardim Tropical e trechos da Vila São José, que são invasões, as outras situam-se em terrenos com intensa cobertura vegetal, igarapés e nascedouros de água, constituindo um ambiente agradável para se viver. Quanto às moradias visitadas, $68,0 \%$ são contruídas de taipa, $48,0 \%$ cobertas de palha e em $66,0 \%$ o piso é de chão batido. Apenas 38,0\% servem-se de água encanada e suas casas não possuem esgotos. Apesar do aspecto pobre, são ventiladas, cercadas de árvores frutíferas, constituindo arruados em meio às plantações, compondo um quadro rural e semi-rural bastante acolhedor. Os animais domésticos mais encontrados são 0 cão e o gato.

Em relação ao tempo de moradia e a situação econômica dos seus habitantes, oitenta por cento moram há mais de um ano e a maioria dos chefes de família não tem emprego fixo e pertence as categorias de lavradores e pescadores, existindo ainda pedreiros, vigilantes, costureiras, aposentados e alguns em atividade de comércio informal. As mulheres executam tarefas domésticas e ajudam a economia do lar cultivando hortas e emprestando sua mão de obra como lavadeiras e arrumadeiras.

\section{DISCUSSÃO}

Durante o trabalho nas localidades e nas visitas domiciliares, surpreendeu a grande migração dos seus habitantes, fato comprovado pelo não encontro de $22,0 \%$ dos pacientes que se mudaram para os limites da própria Ilha ou para o interior do Estado. Ao lado dessa realidade, destaca-se o modo como vivem as famílias, sem o mínimo de assistência à saúde, precário saneamento, tendo até dificuldades financeiras de se deslocarem em busca de assistência para seus enfermos. Foi surpreendente ouvir relatos de mães queixosas cujos filhos foram examinados por vários médicos, sendo internados seguidas vezes com diagnósticos diversos e submetidos a tratamentos variados e inespecíficos.

Estes e outros acontecimentos que contribuem para retardar o tratamento e levar o paciente ao óbito, merecem reflexão de todos os que trabalham no Sistema Único de Saúde. A Medicina sendo uma profissão a serviço do homem e da sociedade 9 obriga eticamente os seus profissionais a utilizarem os recursos disponíveis no sentido de torná-la cada vez mais resolutiva em função da promoção, proteção e recuperação da saúde. Para que tais princípios aconteçam na prática, é necessário o mínimo de organização do sistema de saúde. Tratando-se de calazar, é o município de São Luís que centraliza os recursos para diagnóstico, internação e tratamento.

Em relação às manifestações clínicas dos pacientes acometidos são similares às descrições feitas pelos autores, na seqüência dos clássicos da nossa literatura1 101126 até os atuais7 8141721 . Estes são unânimes em demonstrar que leishmaniose visceral é doença que acomete crianças preferencialmente abaixo dos 9 anos de idade, sendo a faixa etária mais prevalente em nosso meio a compreendida entre 2 a 4 anos. Certamente esta constatação tem a ver com a presença do reservatório (o cão) companheiro do homem com morada dentro ou nos arredores da casa, ao atrair seu transmissor principal a Lutzomya longipalpis3.

O diagnóstico retrospectivo dos casos, valorizou os achados clínicos com destaque para febre arrastada, palidez, emagrecimento e tosse. Também, há referência a protusão abdominal onde o elemento básico do exame físico é o crescimento muitas vezes exagerado do baço e em menor proporção do fígado. A confirmação laboratorial se deu pelo encontro de Leishmania, chamando-se a atenção para o fato de que 44 pacientes, ou seja, 33,0\% dos 130 examinados, tiveram o diagnóstico clínico confirmado pela sorologia. Observa-se que 0 esperado é a alta positividade da reação em presença de doença ativa, tendo-se o cuidado de confirmá-la, enfatizando-se que uma boa anamnese, exame físico bem feito acompanhado de dados epidemiológicos e laboratoriais indiretos ajudam a confirmar a existência do caso de calazar. Isso deve-se ao fato de que, nas áreas onde ocorreram os casos, além de haver pessoas infectadas e não doentes, existem doentes de malária, esquitossomose, hanseníase e outras patologias, que podem dar resultados sorológicos falso positivos 2 .

O tratamento de $96 \%$ dos pacientes foi a base do antimoniato-N-metil-glucamina, droga bem tolerada e de excelentes resultados 72125 27. A outra droga utilizada foi anfotericina B lipossomal em 5 pacientes dos quais dois não responderam, sendo retratados com glucantime com ótimos resultados. Estes 
Silva AR, Viana GMC, Varonil C, Pires B, Nascimento MDSD, Costa JML Leishmaniose visceral (calazar) na Ilha de São Luís, Maranhão, Brasil: evolução e perspectivas. Revista da Sociedade Brasileira de Medicina Tropical 30:359-368, set-out, 1997.

casos não comportam discussão pois fizeram parte de um protocolo de pesquisa em andamento18. Em nosso meio a anfotericina B e a pentamidina são drogas de segunda escolha25.

Barral e cols4, referem a existência de casos de calazar refratários aos antimoniais pentavalentes. No Maranhão, Silva e cols24 25 confirmaram casos com má resposta ao Glucantime ${ }^{\circledR}$, sendo descartados de sua casuística os pacientes que utilizaram subdoses ou abandonaram o tratamento. A Tabela 2 permite constatar que 44 pacientes eram portadores de esplenomegalia. Seguramente $15(34,0 \%)$ com baços palpáveis em graus II e III podem caracterizar a doença em atividade 28 , o que, requer, acompanhamento e confirmação.

Não houve relatos de complicação importante ao uso de Glucantime ${ }^{\circledR}$, embora exista referência na literatura de morte súbita e achados de cardiotoxicidade por antimoniais pentavalentes 6 , droga organodepositária, quando ultrapassa as doses convencionais de até 30mg/Sbv/kg/peso/dia6 19. Font e cols12 relataram a possibilidade de complicações renais e óbitos por coagulação intravascular disseminada. No caso específico do paciente portador de esquitossomose, que foi a óbito 3 horas após a última dose de tratamento, requer cautela, podendo-se apenas registrar, uma vez que não se realizou necropsia.

Quanto ao "critério de cura" que orientou a alta dos pacientes, foi constatado que $66,0 \%$ receberam alta do próprio médico que os trataram. O acompanhamento do paciente, embora nem sempre realizado, é procedimento fundamental ao êxito do tratamento, principalmente no calazar que ainda não tem parâmetros de cura bem estabelecidos. Por esse motivo, somos de acordo que uma boa observação, capaz de acompanhar a regressão da sintomatologia e da esplenomegalia, constitua método para definição de cura. Chamamos a atenção de que a permanência pura e simples do baço após o período de tratamento não é indicativo de prosseguimento do mesmo. Outros dados, como normalização do hemograma, da velocidade de hemossedimentação e do proteinograma poderão servir de parâmetros complementares. Ainda não obtivemos informações suficientes, no acompanhamento após a alta, para avaliar a provável viragem do teste de Montenegro .
Além do interesse da equipe nos casos que foram a óbito, fomos motivados pela população a investigar outros casos de morte. Essa foi a razão pela qual se descobriu mais 9 casos que, após tratamento, faleceram em suas localidades. A Tabela 3 mostra a realidade de óbitos de Calazar em 1994 na Ilha de São Luís, constatando-se que letalidade real foi 67,5 por mil acometidos, o que representa 1,7 vezes mais do que a registrada nas fichas de notificação.

Conclusões. a) constata-se que na vigência do Sistema Unico de Saúde cujos princípios são Descentralização, Universalização do Atendimento, bem como Qualidade e Resolutividade da Assistência, o calazar é uma patologia cuidada de maneira vertical, o que redunda em altos índices de letalidade; b) existe refratariedade ao tratamento com antimoniato-N-metil-glucamina (Glucantime ${ }^{\circledR}$ ) embora essa evidência não comprometa sua eficácia como droga de escolha no tratamento; c) deve-se criar condições concretas para que a rede de serviços através de profissionais de saúde, incluindo os laboratoriais, melhorem 0 diagnóstico de calazar e o mesmo passe a ser responsabilidade do Sistema Único de Saúde em obediência ao que se refere o Artigo 206 da Constituição Brasileira, regulamentado pela Lei 8080/90 e 8142/90; d) há necessidade de estudos que assegurem a verdadeira situação da refratariedade do calazar ao Glucantime ${ }^{\circledR}$ e a outras drogas.

\section{SUMMARY}

Visceral leishmaniasis (kala-azar) was definitively an important disease of the state of Maranhão-Brasil since 1982. Since of then, many authors have been working with this topic in spite of reports. Nevertheless, the aspects of diagnosis, treatment an control of cure went through still hard worried the authors have been studing the disease, that came at São Luís Island since of the desestabilization of the ecotops of Lutzomya longipalpis, most important sandflies bites. After 1993 the constatation of cases with bad response to pentavalent antimonial (Glucantime ${ }^{\circledR}$ ) comes to add the other worries. This actual trial accost the disease and conclude about an existence of failures to Glucantime ${ }^{\circledR}$ being important to have much vigilance in the diagnosis, treatment and control of cure of the patients.

Key-words: Visceral leishmaniasis. Treatment. Clinical aspects. 
Silva AR, Viana GMC, Varonil C, Pires B, Nascimento MDSD, Costa JML Leishmaniose visceral (calazar) na Ilha de São Luís, Maranhão, Brasil: evolução e perspectivas. Revista da Sociedade Brasileira de Medicina Tropical 30:359-368, set-out, 1997.

\section{REFERÊNCIAS BIBLIOGRÁFICAS}

1. Alencar JE, Aragão T. Leishmaniose visceral no Ceará. Sintomas observados em 174 casos. Diagnóstico clínico. Apresentado ao XII Congresso Brasileiro de Higiene. Belém, PA, 1955.

2. Alencar JE, Neves J, Dietze R. Leishmaniose visceral (calazar). In: Veronesi R (ed) Doenças Infecciosas e Parasitárias. 8a Edição. Guanabara Koogan, Rio de Janeiro, I99l.

3. Alvim MC, Soares OR, Castro Gomes CM, Ferreira MIS. Levantamento da fauna flebotomínica da Ilha de São Luís I. Espécies antropofilicas. In: Resumos do XXV Congresso da Sociedade Brasileira de Medicina Tropical, Florianópolis, p.53, 1989.

4. Barral A, Barral-Neto M. Avaliação da resistência as drogas na leishmaniose. Revista da Sociedade Brasileira de Medicina Tropical 27 (supl I):251, 1994.

5. Berhe N, Ali A, Hailu A, Yeneneh H. Relapse in Ethiopian Visceral Leishmaniasis (VL) patients after therapy with pentavalent antimonials: a ten years observation. Institute of Pathobiology (IPB), AddisAbeba University (AAU), Ethiopia. Annals Tropical Medicine and Parasitology 88:289-293, 1993.

6. Chulay JD, Spencer HC, Muganbi M. Electrocardiographic changes during treatment of leishmaniases with pentavalent antimony (sodium stiboglucanate). The American Journal of Tropical Medicine and Higiene 34:702-709, 1985.

7. Costa CHN, Pereira HF, Araujo MV. Epidemia de leishmaniose visceral no Estado do Piauí, Brasil, 1980-1986. Revista de Saúde Pública 24:361-371, 1990.

8. Costa JM L, Viana GMC, Sal danha ACR, N ascimento MDS, Alvim AC, Burattini MN, Silva AR. Leishmaniose visceral no Estado do Maranhão, Brasil. Evolução de uma epidemia. Cadernos de Saúde Pública 11:32I-24, 1995.

9. Conselho Federal de Medicina. Código de Ética Médica. Resolução CFM, no 1246/88. Rio de Janeiro, 1988.

10. Deane L, Deane GM. Estudo sobre leishmaniose visceral americana. Nota $\mathrm{n}-1$. Aspectos clínicos da doença. O Hospital I2:189-199, 1937.

11. Fiquene SF. Analogias parasitárias, novo tratamento de leishmaniose. Revista Maranhão Médico 2:9-18,1964.

12. Font A, Gines C, Closa JM, Mascort J. Visceral leishmaniases and disseminated intavascular coagulation in a dog. Hospital Ars Veterinaria, Barcelona, Spain. Journal American Veterinary Medical Association 204: 1043-1044, 1994.

13. Hackett LW. Spleen measurement in Malaria. Journal National Malaria Society 3:121-33, 1944.

14. Marzochi KBF, Marzochi MCA, Andrade MV, Silva VL, Gomes MZR, Silveira MIF, Almeida DC. Avaliação prospectiva da leishmaniose visceral autóctone no Município do Rio de Janeiro. I- Dados clínicos e laboratoriais. Revista da Sociedade Brasileira de Medicina Tropical 27 (supl I): 257, 1994.

15. Monteiro PS, Lacerda MM, Arias JR. Controle da leishmaniose visceral no Brasil. Revista da Sociedade Brasileira de Medicina Tropical 27 (supl III): 67-72,1994.

16. Nascimento MDSB, Costa JML, Fiori BIP, Viana GMC, Filho MSG, Alvim AC, Bastos OC, Nakatani $M$, Reed S, Badaró R, Silva AR, Burattini MN. Aspectos epidemiológicos determinantes na manutenção da leishmaniose visceral no Estado do Maranhão-Brasil. Revista da Sociedade Brasileira de MedicinaTropical 29:233-240, 1996.

17. Nascimento MDSB, Fiori BIP, Carneiro LS, Gonçalves Filho M, Buratini MN. Estado atual da leishmaniose visceral no Maranhão: aspectos epidemiológicos preliminares. Revista da Sociedade Brasileira de Medicina Tropical 27 (supl I):242, 1994.

18. Saldanha ACR, Alvim MAB, Alvim AC, Balby ITA, Nascimento JDL, Viana GMC, Carneiro CS, Costa JML. Treatment of visceral leishmaniasis with lipid-associated amphotericin B (ambisome) in the state of Maranhão. Preliminary report. Revista da Sociedade Brasileira de Medicina Tropical 27 (supl I):93,1994.

19. Sampaio RNR, N etto EM, Faria EA, Sampaio JHD, Freitas LCF, Marsden PD. Morte súbita causada por Glucantime ${ }^{\circledR}$. Anais Brasileiros de Dermatologia 63:35-37,1988.

20. Silva AR. Malária. Fotografia de uma crise no setor saúde. Maranhão, Editora da Universidade Federal do Maranhão, São Luís, 1989.

21. Silva AR, Brasil R, Pedroso e Silva CM, Branco MRC, LeiteW. Aspectos epidemiológicos, clínicos e terapêuticos da leishmaniose visceral (calazar) na llha de São Luís, Estado do Maranhão-Brasil de 1982 a 1987. In: Resumos do XXIV Congresso da Sociedade Brasileira de MedicinaTropical, Manaus, p.114, 1988. 
Silva AR, Viana GMC, Varonil C, Pires B, Nascimento MDSD, Costa JML Leishmaniose visceral (calazar) na Ilha de São Luís, Maranhão, Brasil: evolução e perspectivas. Revista da Sociedade Brasileira de Medicina Tropical 30:359-368, set-out, 1997.

22. Silva AR, Costa JM, Mochel A, Carneiro EWB, Brasil R. Leishmaniose visceral na Ilha de São Luís, Estado do Maranhão. I. Aspectos clínicos e terapêuticos. In: Resumos do XIX Congresso de Sociedade Brasileira de MedicinaTropical. Rio de Janeiro, p.65, 1983.

23. Silva AR, Viana GMC, Costa GSL, Lindoso JAL. Aspectos do calazar na lha de São Luís de 1988 a 1992. In: Resumos do XXIX Congresso da Sociedade Brasileira de MedicinaTropical, Fortaleza, p.230, 1993.

24. Silva AR, Viana GMC, Nascimento MDSB, Costa JML. Leishmaniose visceral (calazar) na llha de São Luís-MA, anos depois. In: Resumos do XXXII Congresso da Sociedade Brasileira de Medicina Tropical. Goiânia, p.148, 1996.
25. Silva AR, Viana GMC, Varonil C, Pires B. É a Nmetilglucamina (Glucantime ${ }^{\circledR}$ ) arma importante no controle da leishmaniose visceral (calazar) em nosso meio? In: Resumos da 47a Reunião da Sociedade Brasileira para o Progresso da Ciência (SBPC). São Luís, p.78, 1995.

26. Silva JR. Leishmaniose visceral (calazar). Publicação do Serviço Nacional de Educação Sanitária. Rio de Janeiro, RJ, 1956.

27. Viana GM, Costa JML, Silva AR. Leishmaniose visceral no Maranhão 1960 a 1993. Revista da Sociedade Brasilleira de Medicina Tropical 27 (supl I):240, 1994.

28. World Health Organization. Leishmanaiasis. Geneva, WHO, 151 p. (Technical Report Series 793), 1984. 